\title{
Hacia una propuesta para evaluar ambientes virtuales de aprendizaje (AVA) en Educación Superior ${ }^{1}$
}

\author{
Erika Juliana Estrada Villa² \& Oscar Rafael Boude Figueredo ${ }^{3}$ \\ Universidad de La Sabana, Colombia
}

Recibido, abril 15 de 2015

Concepto evaluación, junio 29 de 2015

Aceptado, octubre 15 de 2015
Referencia: Estrada Villa, E.; Boude Figueredo, O. (2015). “Hacia una propuesta para evaluar ambientes virtuales de aprendizaje (AVA) en Educación Superior". Revista Academia y Virtualidad, $8,(2), 14-23$

\section{Resumen}

Este artículo tiene como propósito presentar un instrumento para evaluar un ambiente virtual de aprendizaje (AVA). El documento se basa en primera instancia en los conceptos de ambiente de aprendizaje; en segundo lugar, en la evaluación, considerando los principales aspectos que lo conforman. El análisis es complementado con el modelo propuesto para evaluar el AVA con base en una rúbrica de siete criterios de evaluación que aborda tanto la intención pedagógica como la interacción de la tecnología, y finaliza con la aplicación de la rúbrica en un proceso de autoevaluación al ambiente de aprendizaje realizado e implementado durante la práctica I y II de la Maestría en Informática Educativa de la Universidad de La Sabana en 2013. En esta reflexión se presenta un instrumento de evaluación, basado en los modelos y teorías de la evaluación educativa como punto de partida para la evaluación de ambientes virtuales de aprendizaje en programas no presenciales en Educación Superior en Colombia.

Palabras clave: ambientes de aprendizaje; evaluación educativa; rúbrica; TIC.

\section{A proposal to assess virtual learning environments -VLE- in higher education}

\section{Abstract}

This paper aims to give a rubric to assess a virtual learning environment-VLE. Firstly, it is based upon learning environment concepts; secondly, we use assessment issues taking into account its main sides. This analysis is supplemented with a model to assess a VLE based on a 7-criteria rubric addressing both the teaching goal and a

1. Artículo de carácter reflexivo que aporta una solución para evaluar ambientes virtuales de aprendizaje, como resultado del trabajo de investigación del Proyecto Profesoral Observatorio Informática Educativa del Grupo de Investigación Proventus del Centro de Tecnologías para la Academia de la Universidad de La Sabana.

2. Magíster en Informática Educativa de la Universidad de La Sabana. Docente y asesora de Investigación de la Escuela de Postgrados. Asesora Trabajos de Grado Maestría en Informática Educativa Centro de Tecnologías para la Academia Universidad de la Sabana. Correo: erikajulianaestrada@hotmail.com

3. Doctor en ciencias de la educación; profesor-investigador Maestría en Informática Educativa Centro de Tecnologías para la Academia Universidad de La Sabana. Correo: oscar.boude@unisabana.edu.co 
technology combination but finally implementing the rubric in a self-assessment of the learning environment carried out and implemented during practice I \& II of the Educational Computing Master at Universidad de La Sabana during 2013. This reflection offers an assessment tool based on models and theories of educational assessment as a starting point for evaluation of virtual learning environments in non-face programs of Colombian higher education.

Keywords: learning environments, educational assessment, rubric, ICT.

\section{Em direção a uma proposta para a avaliação de ambientes de aprendizado (AVA) na Educação superior}

\section{Resumo}

Este artigo tem como propósito apresentar um instrumento para a avaliação dum ambiente virtual de aprendizado (AVA). O documento baseia-se na primeira instancia nos conceitos de ambiente de aprendizado; em segundo lugar, na avaliação, considerando os principais aspetos que a conformam. A análise é complementada com o modelo proposto para avaliar AVA baseada numa rubrica de sete critérios de avaliação que aborda tanto a intensão pedagógica quanto a interação da tecnologia, e finaliza com a aplicação da rubrica num processo de auto avaliação ao ambiente de aprendizagem realizado e operacionalizado durante a prática I e II da Mestria em Informática Educativa da Universidade da Sabana em 2013. Nesta reflexão apresenta-se um instrumento de avaliação, baseado nos modelos e teorias de avaliação educativa como ponto de partida para a avaliação de ambientes virtuais de aprendizado em programas não presenciais na Educação Superior na Colômbia.

Palavras chave: ambientes de aprendizado, avaliação educativa, rubrica, TIC.

\section{Introducción}

El presente artículo se realiza dentro de la investigación de maestría Factores que facilitan y dificultan la inclusión del aprendizaje móvil en Educación Superior, dentro del proyecto profesoral Observatorio de Informática Educativa, registrado en el grupo de investigación Proventus del Centro de Tecnologías para la Academia de la Universidad de La Sabana; y como parte de la implementación del ambiente virtual de aprendizaje en cumplimiento de la línea de énfasis "Integración de las TIC en ambientes de aprendizaje", y para intervenir al factor capacitación en dicha investigación.

Este ejercicio investigativo parte de una revisión de los teóricos de la evaluación educativa y desde el análisis de los componentes de los ambientes de aprendizaje.
Asimismo, se justifica en la necesidad de contextualizar a los docentes en los docentes en la evaluación de ambientes virtuales de aprendizaje dentro de la revolución tecnológica que se está viviendo actualmente.

A través de los conceptos en un recorrido histórico sobre la evaluación educativa y de los elementos de los ambientes de aprendizaje, para llegar a proponer una rúbrica a fin de evaluar ambientes virtuales de aprendizaje (AVA), la cual se pone en práctica a través de un ejemplo, y se finaliza con una reflexión acerca de los principales criterios por tener en cuenta a la hora de evaluar, lo que permite tener una visión más amplia de los que se está gestando en el proceso de aprendizaje en este tipo de escenarios. Obteniendo como conclusión que la evaluación educativa es un instrumento importante para el mejoramiento continuo en busca de la calidad del proceso enseñanza- 
Hacia una propuesta para evaluar ambientes virtuales de aprendizaje (AVA) en Educación Superior

aprendizaje de los ambientes virtuales de aprendizaje, a partir de la interpretación y análisis de los aspectos evaluados.

\section{Conceptos abordados}

Para comprender qué es evaluación, es importante mencionar que la evaluación educativa surge a principios del siglo XX en la década de los 40 con las teorías de Ralph Tyler; posteriormente, en la década de los 60, se reconceptualiza con teorías de autores como Cronbach, Glaser, Eisner, Scriven y Stake, donde la evaluación se plantea como un proceso sistemático para analizar, interpretar y emitir juicios de valor respecto a una información específica producto de un proceso educativo con el fin de identificar y medir la calidad, las cualidades y la eficacia de un programa, proyecto o método para permitir la toma de decisiones en busca de un mejoramiento continuo (Lukas, 2009).

El propósito de la evaluación del AVA es más que un medio para juzgar, es un proceso metódico que analiza e interpreta la información recolectada para la toma de decisiones en cuanto a los diferentes aspectos que lo componen, con el propósito de mejorar el objeto de evaluación. Para adentrarnos en el tema es necesario conocer la definición de ambiente de aprendizaje así como de sus componentes A continuación se aborda este aspecto.

\section{Ambientes virtuales de aprendizaje}

Boude (2011) define un ambiente de aprendizaje como un espacio virtual o presencial diseñado e implementado por el profesor con la clara intención de contribuir al desarrollo de competencias y habilidades en sus estudiantes, lo que implica por parte de éste seguir un proceso reflexivo en el que a partir del reconocimiento del contexto educativo se dan respuestas sobre qué, cómo y para qué se enseña. En el ambiente de aprendizaje intervienen los estudiantes, el profesor y el currículo. Un ambiente de aprendizaje está concebido para solucionar problemas como falta de comprensión, indisciplina, problemas de aprendizaje, entre otros factores comunes en el escenario pedagógico. Para Otálora (2010), un ambiente de aprendizaje constituye un escenario de construcción de conocimiento en el que un agente educativo -o institución educativa, organización o grupo cultural- genera intencionalmente un conjunto de actividades y acciones dirigidas a garantizar la consecución de un objetivo de aprendizaje amplio, que es pertinente para el desarrollo de competencias en uno o varios dominios de conocimiento de uno o más educandos pertenecientes a una cultura. El concepto de ambiente de aprendizaje no sólo se refiere a la totalidad de las actividades que giran alrededor de un objetivo de aprendizaje centrado en un conocimiento específico, un contenido temático o una habilidad, como tradicionalmente se concibe. Un ambiente de aprendizaje es un espacio estructurado en el que se articulan diversos elementos y relaciones necesarios para alcanzar tal objetivo.

En el documento Informe mundial sobre el aprendizaje y la educación de adultos, la Unesco (2010) menciona que los entornos de aprendizaje virtuales constituyen una forma totalmente nueva de tecnología educativa y ofrece una compleja serie de oportunidades y tareas a las instituciones educativas de todo el mundo, y define al ambiente de aprendizaje como un programa informático interactivo de carácter pedagógico que posee una capacidad de comunicación integrada, es decir, que está asociado a las nuevas tecnologías de la información y están favorecidos por la incorporación de las tecnologías donde se potencia la educación virtual por estar basado en la educación no presencial y donde el centro es el aprendiz.

Vista la definición de ambientes virtuales de aprendizaje, con la información de la figura 1 se abordan los componentes del mismo que van desde la intención pedagógica hasta las evidencias del proceso de aprendizaje; dichos componentes serán los criterios y parámetros de evaluación de la rúbrica AVA (ver tabla 2). 
Erika Juliana Estrada Villa \& Oscar Rafael Boude Figueredo

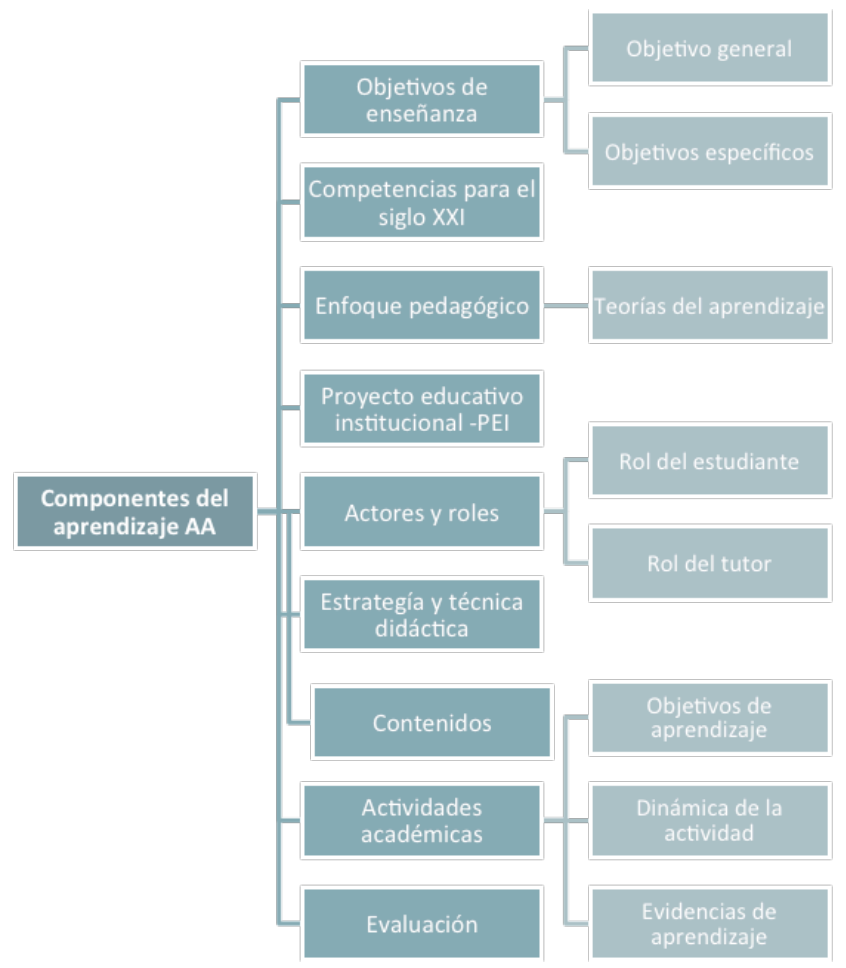

Figura 1. Esquema de los componentes del ambiente virtual de aprendizaje.

Conociendo los parámetros sobre los cuales se diseñó la rúbrica AVA y según lo indicado por Lukas (2009) quien menciona que al momento de evaluar es fundamental tener claridad sobre el objetivo que persigue la evaluación, paso seguido se exponen los componentes para evaluar el AVA.

\section{Componentes de la evaluación}

La evaluación es un proceso con diferentes funciones que permite diagnosticar el estado y el alcance del ambiente virtual de aprendizaje. La evaluación comprueba el grado de enseñanza-aprendizaje, además de servir como medio de comunicación entre el estudiante y el docente y de ejercicio de retroalimentación para la mejora continua del proceso educativo.

A través de la observación sistemática que se da en un proceso de evaluación se pueden detectar e intervenir los problemas que se perciben en el ambiente virtual de aprendizaje. A través de la evaluación se genera un enfoque holístico a la percepción que se tiene del ambiente de aprendizaje, teniendo en cuenta los múltiples factores que intervienen en éste (Cabero, 2002).

Asimismo, se debe tener en cuenta que la evaluación aporta interpretaciones al ambiente de aprendizaje toda vez que en el proceso de evaluación existe un continuo contraste que le permiten al evaluador realizar un proceso cíclico en donde puede analizar, comparar, aplicar, integrar y, de esta forma, lograr la comprensión de la observación sistemática del AVA que va de lo particular hasta lo general, generando así lo que se conoce como un proceso inductivo de conocimiento (Lukas, 2009).

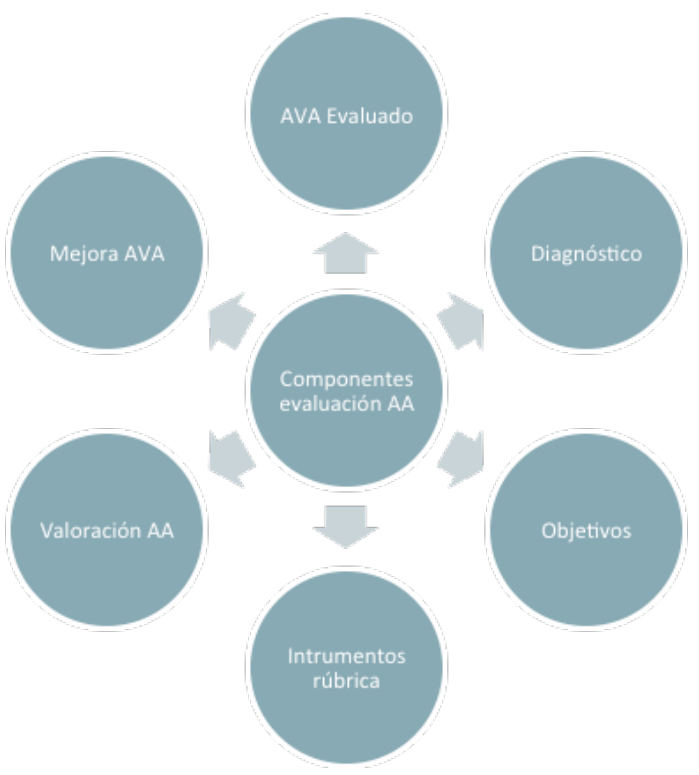

Figura 2. Componentes de la evaluación de ambientes virtual de aprendizaje

El objetivo principal de la evaluación del ambiente virtual de aprendizaje es comprender y analizar si los objetivos trazados se lograron, y para esto se deben tener en cuenta los componentes que se relacionan en la evaluación del ambiente virtual de aprendizaje (ver figura 2) y se relacionan a continuación:

I. AVA evaluado: constituye el actor principal del proceso de evaluación

II. Diagnóstico inicial: se debe tener en cuenta las necesidades y problemáticas, debilidades y fortalezas del AVA y de sus participantes. 
III.Objetivos: Indican qué hacer, cómo hacer y el para qué se hace.

IV. Instrumentos - Rúbrica: Instrumento o método empleado y diseñado, y son vistos como las fuentes de información que se emplearán para el cumplimiento de los objetivos de la evaluación.

V. Valoración: después de aplicar los instrumentos y obtener la información se tienen los elementos para realizar la valoración del objeto.

VI. Mejoramiento AVA: después de tener la valoración a través del proceso de evaluación, se realizan los ajustes mejoramiento, si los hubiese, al objeto evaluado.

Los componentes descritos anteriormente son tomados a manera de ruta para la construcción y aplicación de la rúbrica AVA, es necesario tener en cuenta que esta propuesta es para evaluar el diseño del AVA y no el impacto o la suficiencia que tuvo el AVA en sus participantes. Básicamente lo que se realiza en la evaluación de un AVA es la recolección de información que permite al evaluador aproximarse a una realidad específica y explicar una o varias situaciones que ocurren al interior de éste. Así, el evaluador parte de la realidad analizada y puede convertir dicha realidad en resultados que explican el porqué suceden dichos fenómenos y cuáles son las causas, para así proponer posibles soluciones o herramientas a incluir en los planes de mejora.

Después de conocer las definiciones de ambiente de aprendizaje y de evaluación, y descritos todos los componentes para estructurar el instrumento tratado en el presente documento, a continuación se da paso a exponer la propuesta para la evaluación de ambientes virtuales de aprendizaje.

\section{Propuesta de evaluación para ambientes virtuales de aprendizaje}

Para la evaluación del ambiente virtual de aprendizaje se propone una rúbrica que aborda siete criterios de evaluación y a su vez cada uno de éstos agrupa cinco parámetros (ver tabla 1). Los principales criterios, los cuales se identifican con las letras de la A hasta la G, se establecen a partir de las definiciones abordadas en la sección anterior, además de vincular elementos del diseño de ambientes de aprendizaje propuesto por Boude (2012). Para el criterio F: integración de las TIC, se retoman los parámetros de evaluación propuestos por Harris, Grandgenett \& Hofer (2010) en su rúbrica de evaluación de integración de tecnología. Los parámetros de evaluación del criterio G: calidad y pertinencia, son adaptados del instrumento para la evaluación de materiales multimedia propuestos por Cerrano, Fulgueira, \& Gómez (sf).

Teniendo en cuenta lo anterior para evaluar el ambiente virtual de aprendizaje se abordarán los siguientes aspectos: el planteamiento de los objetivos de enseñanza y que proponga como mínimo una competencia para el siglo XXI propuestas por la UNESCO; que las teorías sobre las cuales se fundamenta el AVA estén acordes con el proyecto educativo institucional de la institución. Debe indicar asimismo los roles del estudiantes y del tutor con actividades definidas. Las estrategias y técnicas didácticas deben estar alineadas con el contexto y se deben identificar en los contenidos. Se examinará de otra parte que las actividades académicas deben contemplar el objetivo de aprendizaje, la dinámica para su desarrollo y su correspondiente evaluación. El AVA debe evidenciar la integración de las tecnologías de la información y de la comunicación. Por último, se espera evaluar la calidad y la pertinencia del ambiente propuesto.

La propuesta para la evaluación de ambientes virtuales de aprendizaje aquí planteada vincula diversos criterios de evaluación de diferentes autores, para que en un futuro sirva de guía para la elaboración de ambientes de aprendizaje. La rúbrica para la evaluación de ambientes de aprendizaje no mide el grado de conocimiento ni de destreza que puede tener quien lo diseña, está diseñado para no olvidar y tener presente los aspectos esenciales a la hora de diseñar un ambiente de aprendizaje. 
Erika Juliana Estrada Villa \& Oscar Rafael Boude Figueredo

Rúbrica AVA

Título AVA:

Nombre:

Instrucciones: Asigne los puntos a cada parámetro en la columna derecha. Evalúe I-

4 siendo 4 (cuatro) la nota más alta y I (uno) la más baja

\begin{tabular}{|l|l|l|l|l|} 
Siempre & Casi siempre & A veces & Casi nunca & Nunca
\end{tabular}

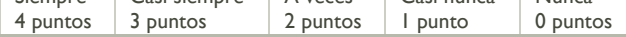

A: Objetivos de enseñanza y competencias para el siglo XXI El AVA posee un objetivo y una intención pedagógica clara

El objetivo del AVA se expresa en el contenido

El objetivo está alineado con el contexto y las competencias

El AVA propone el desarrollo de alguna competencia para el siglo XX

Las competencias están articuladas con las actividades académicas

5 (20-18) / 4 (17-14) / 3 (13-9) / 2 (8-5) / I (I-5)

\begin{tabular}{|l|l|}
\hline \multicolumn{1}{|c|}{ B: Enfoque pedagógico (teorías del aprendizaje) } & Puntos \\
\hline El AVA se fundamenta en un enfoque de aprendizaje & \\
\hline El enfoque de aprendizaje se evidencia en las actividades académicas del AVA & \\
\hline El AVA es pertinente al PEI de la institución educativa & \\
\hline El AVA facilita el aprendizaje significativo y contiene una actividad que lo potencia & \\
\hline El AVA promueve el aprendizaje por descubrimiento & \\
\hline $5(20-18) / 4(17-14) / 3(13-9) / 2(8-5) / I(I-5)$ & \\
\hline
\end{tabular}

\begin{tabular}{|l|l|}
\hline \multicolumn{1}{|c|}{ C: Actores y comunicación } & Puntos \\
\hline El AVA evidencia las funciones y roles de los actores & \\
\hline EL AVA posee y evidencia el rol del estudiante & \\
\hline EL AVA posee y evidencia el rol del tutor & \\
\hline EI AVA favorece la comunicación entre los participantes & \\
\hline El AVA tiene un lenguaje académico adecuado a los estudiantes y al tema & \\
\hline $5(20-18) / 4(17-14) / 3(13-9) / 2(8-5) / 1(1-5)$ & \\
\hline
\end{tabular}

\begin{tabular}{|l|l|}
\hline \multicolumn{1}{|c|}{ D: Estrategia y contenidos } & Puntos \\
\hline El AVA está basado en una estrategia y técnica didáctica & \\
\hline EL AVA posee una secuencia didáctica y coherencia con la intención pedagógica & \\
\hline Es pertinente la temática al nivel educativo & \\
\hline Los temas centrales tienen más relevancia dentro del AVA y están destacados & \\
\hline Los contenidos del AVA corresponden al contenido programático & \\
\hline $5(20-18) / 4(17-14) / 3(13-9) / 2(8-5) / I(I-5)$ & \\
\hline
\end{tabular}

Observaciones

EJE 2013

\begin{tabular}{|l|l|}
\hline \multicolumn{1}{|c|}{ E: Actividades académicas y evaluación } & Puntos \\
\hline Las actividades académicas del AVA mencionan el objetivo de aprendizaje & \\
\hline $\begin{array}{l}\text { EI AVA menciona la dinámica de la actividad, poseen una conducta de entrada e instrucciones } \\
\text { claras para su desarrollo }\end{array}$ & \\
\hline $\begin{array}{l}\text { El AVA menciona cómo se van a evaluar las actividades académicas y corresponden a los } \\
\text { contenidos y al material diseñado para el curso }\end{array}$ & \\
\hline La evaluación es coherente con el contenido del curso & \\
\hline El AVA utiliza elementos que promueven la autoevaluación, coevaluación y heteroevaluación & \\
\hline $5(20-18) / 4(17-14) / 3(13-9) / 2(8-5) / I(I-5)$ & \\
\hline
\end{tabular}

\begin{tabular}{|l|l|}
\hline \multicolumn{1}{|c|}{ F: Integración de las TIC } & Puntos \\
\hline Los recursos educativos utilizados justifican la enseñanza del tema & \\
\hline El uso de la tecnología apoya de manera óptima las estrategias de enseñanza (Harris, 20I0) & \\
\hline $\begin{array}{l}\text { La selección de tecnologia(s) es ejemplar con respecto a los objetivos curriculares y las } \\
\text { estrategias de enseñanza (Harris, 20/0) }\end{array}$ & \\
\hline El uso de la tecnología apoya de manera óptima las estrategias de enseñanza (Harris, 20I0) & \\
\hline $\begin{array}{l}\text { Contenido, estrategias de enseñanza y tecnología se articulan completamente entre sí dentro } \\
\text { de la planificación didáctica (Harris, 20/0) }\end{array}$ & \\
\hline $5(20-18) / 4(17-14) / 3(13-9) / 2(8-5) /$ / (I-5) & \\
\hline
\end{tabular}

$5(20-18) / 4(17-14) / 3(13-9) / 2(8-5) / 1(1-5)$

\section{G: Calidad y pertinencia}

Aspectos técnicos del AVA (basado en Cerrano, et al (sf).)

Distribución y calidad de contenidos (basado en Cerrano, et al (sf).)

Aspectos pedagógicos y motivacionales (basado en Cerrano, et al (sf).)

Valoración global y contextual del AVA (basado en Cerrano, et al (sf).)

El AVA maneja adecuadamente los derechos de autor

5 (20-18) / 4 (17-14) / 3 (13-9) / 2 (8-5) / I (I-5)

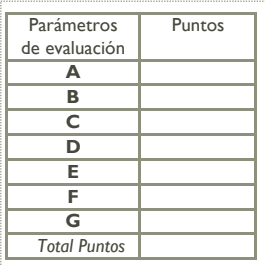

Observaciones

EJE 2013

Tabla 1. Rúbrica propuesta para la evaluación de ambientes virtuales de aprendizaje

Presentado el instrumento con el cual se va evaluar el AVA se hace pertinente describir los aspectos generales y estructura para tener elementos a la hora de aplicar la rúbrica, a continuación se describen éstos a través de una aplicación práctica.

\section{Piloto: descripción del ambiente virtual de aprendizaje a evaluar con la rúbrica $A V A$}

El ambiente virtual de aprendizaje propone los conceptos básicos del M-Learning a través de una perspectiva del aprendizaje significativo, con el objetivo de generar un sentido de apropiación sobre las prácticas docentes que involucren los dispositivos móviles en las experiencias de enseñanza y aprendizaje con el uso pedagógico de las TIC. En la tabla 2 se presentan brevemente sus principales aspectos. 
Hacia una propuesta para evaluar ambientes virtuales de aprendizaje (AVA) en Educación Superior

\begin{tabular}{|c|c|}
\hline Título $A V A$ & $\begin{array}{l}\text { Ambiente de aprendizaje para vincular el M-Learning a procesos de } \\
\text { enseñanza-aprendizaje en instituciones de Educación Superior }\end{array}$ \\
\hline Profesor & Erika J. Estrada V. \\
\hline $\begin{array}{l}\text { Nombre } \\
\text { Curso }\end{array}$ & M-Learning \& Educación Superior \\
\hline $\begin{array}{l}\text { Marco } \\
\text { temporal y } \\
\text { duración }\end{array}$ & $\begin{array}{l}\text { Ciclo introductorio. Total: } 30 \text { horas virtuales de trabajo independiente. } \\
\text { Entre el } 18 \text { de septiembre y el } 18 \text { de octubre de } 2013\end{array}$ \\
\hline Aula virtual & Virtual Sabana - Curso M-Learning \& Educación Superior \\
\hline Contexto & $\begin{array}{l}\text { Esta estrategia está dirigida a los docentes universitarios de diferentes } \\
\text { disciplinas de instituciones de Educación Superior en Colombia que quieran o } \\
\text { necesiten desarrollar las competencias de innovación y creatividad para } \\
\text { vincular los dispositivos móviles a sus actividades académicas. }\end{array}$ \\
\hline Alcance & $\begin{array}{l}\text { En esta estrategia sólo llegamos hasta que los estudiantes realicen el diseño de } \\
\text { una actividad académica que proponen para vincular el aprendizaje móvil en } \\
\text { su syllabus. }\end{array}$ \\
\hline $\begin{array}{l}\text { Rol del } \\
\text { Docente: }\end{array}$ & $\begin{array}{l}\text { Profesional y/o instructor que diseña, planea, orienta, ejecuta y evalúa el } \\
\text { ambiente de aprendizaje para el uso de dispositivos móviles en Educación } \\
\text { Superior así como diseñar las estrategias didácticas acorde con cada una de las } \\
\text { actividades y aplicaciones para dispositivos móviles. }\end{array}$ \\
\hline $\begin{array}{l}\text { Rol del } \\
\text { Estudiantes: }\end{array}$ & $\begin{array}{l}\text { Cumplir con las aplicaciones prácticas y contextualizadas en el uso de las } \\
\text { diferentes aplicaciones para dispositivos móviles. Así como integrar e incluir } \\
\text { el M-Learning dentro de los procesos de enseñanza-aprendizaje de cada uno } \\
\text { para propiciar mecanismos y estrategias de conectividad entre los estudiantes } \\
\text { y con el docente }\end{array}$ \\
\hline $\begin{array}{l}\text { Descripción } \\
\text { de la } \\
\text { Estrategia }\end{array}$ & $\begin{array}{l}\text { Esta estrategia propone vincular dispositivos móviles en las actividades } \\
\text { académicas universitarias. La estrategia se plantea en cuatro módulos que el } \\
\text { estudiante debe seguir para que integre los dispositivos móviles, reconociendo } \\
\text { las características del aprendizaje móvil, es decir, aquella fundamentación y } \\
\text { modelos pedagógicos que lo diferencian del resto de procesos educativos, los } \\
\text { tipos de usuarios y los usos que éstos le dan a los dispositivos, así como } \\
\text { reconocer la forma en que se da el proceso de comunicación entre estudiantes } \\
\text { y entre el docente y sus estudiantes. En un segundo momento, el ambiente de } \\
\text { aprendizaje lo gúa a integrar e incluir el aprendizaje móvil dentro de los } \\
\text { procesos de enseñanza-aprendizaje a través de la práctica, de tal manera que } \\
\text { pueda determinar la mejor forma de innovar y de generar sus propias } \\
\text { actividades a través del aprendizaje móvil. }\end{array}$ \\
\hline Contenido & $\begin{array}{l}\text { Módulo 1. Introducción } \\
\text { Módulo 2. Fundamentos del aprendizaje móvil } \\
\text { Módulo 3: Experiencias de aprendizaje móvil } \\
\text { Módulo 4: Estrategia } \\
\text { Módulo 5: Cierre y evaluación }\end{array}$ \\
\hline
\end{tabular}

Tabla 2. Descripción y aspectos generales del ambiente virtual de aprendizaje por evaluar

\section{Aplicación de la Rúbrica AVA al ambiente virtual de aprendizaje}

Dentro de la investigación se implementó el "Ambiente de aprendizaje para vincular el M-Learning a procesos de enseñanza-aprendizaje en instituciones de Educación Superior" que contiene la "Estrategia para el desarrollo de las competencias de Innovación y creatividad en docentes universitarios enfocado al aprendizaje móvill", fundamentado en el "Modelo estrategias de aprendizaje móvil”, propuesto por Boude \& Jiménez (2016, en prensa), evidenciado en Virtual Sabana en el curso "M-Learning \& Educación Superior", orientado en cinco sesiones virtuales, durante los meses de septiembre y octubre de 2013 a 30 profesores universitarios. Con el objetivo de contribuir a la apropiación de conceptos básicos sobre el
M-Learning, así como el diseño de actividades estrategias de aprendizaje móvil que fortalezcan los procesos de enseñanza universitaria. Para el desarrollo del AVA fue necesario contar con el apoyo técnico y académico del CTA de la Universidad de la Sabana, así como el aula virtual en la plataforma moodle en Virtual Sabana.

A continuación, en la tabla 3, se presenta la evaluación del ambiente virtual de aprendizaje realizada por un par. De la aplicación de la rúbrica se evidencia que el enfoque pedagógico del AVA se basa en el aprendizaje significativo, que se resalta en las actividades académicas.

Asimismo, se examina el desarrollo de la estrategia y técnica didáctica relacionando en diversas actividades como foros, presentaciones, códigos QR, entre otros recursos y herramientas del ambiente virtual de aprendizaje. Los recursos allí propuestos llevan a que el estudiante explore el aprendizaje móvil en su entorno para diseñar actividades académicas, potencializando sus conocimientos previos. En el aula virtual se destacan los contenidos de mayor relevancia con animaciones en el encabezado.

\section{Resultados}

De la aplicación de la rúbrica "Ambiente de aprendizaje para vincular el M-Learning a procesos de enseñanzaaprendizaje en instituciones de Educación Superior", se destaca que las temáticas se integran y corresponden tanto al contenido programático como al aprendizaje móvil. El material publicado respeta las normas de autor y sigue las reglas básicas de ortografía, gramáticas y manejo de las mayúsculas.

Las actividades permiten la selección de procedimientos, la autoevaluación así como la reflexión de la práctica docente de quien lo realiza. Una de las ventajas que arrojó el AVA diseñado es que es transversal a cualquier área del conocimiento; por ser un curso para la integración del M-Learning, permitió a los participantes diseñar actividades para la integración del aprendizaje móvil en su práctica docente. Las evaluaciones planteadas corresponden a los temas vistos y al material diseñado 
Erika Juliana Estrada Villa \& Oscar Rafael Boude Figueredo

Rúbrica evaluación AVA

Fecha: $25 / 11 / 2013$

Título AVA: Ambiente de aprendizaje para vincular El M-Learning a procesos de enseñanza-aprendizaje en instituciones de Educación Superior

Nombre: Erika J. Estrada V.

Instrucciones: Asigne los puntos a cada parámetro en la columna derecha. Evalúe I4 siendo 4 (cuatro) la nota más alta y I (uno) la más baja

\begin{tabular}{|l|l|l|l|l|}
\hline Siempre & Casi Siempre & A veces & Casi nunca & Nunca \\
4 puntos & 3 puntos & 2 puntos & I punto & 0 puntos \\
\hline
\end{tabular}

A: Objetivos de enseñanza y competencias para el siglo XXI El AVA posee un objetivo y una intención pedagógica clara

El objetivo del AVA se expresa en el contenido

El objetivo está alineado con el contexto y las competencias

El AVA propone el desarrollo de alguna competencia para el siglo XXI

Las competencias están articuladas con las actividades académicas

$5(20-18) / 4(17-14) / 3(13-9) / 2(8-5) / 1$ (I-5)

B: Enfoque pedagógico (teorías del aprendizaje) El AVA se fundamenta en un enfoque de aprendizaje

El enfoque de aprendizaje se evidencia en las actividades académicas del AVA

El AVA es pertinente al $\square E I$ de la institución educativa

El AVA facilita el aprendizaje significativo y contiene una actividad que lo potencia

El AVA promueve el aprendizaje por descubrimiento

5 (20-18) / 4 (I7-14) / 3 (13-9)/ 2 (8-5)/ I (I-5)

\section{$\square$ : Actores y comunicación}

EI AVA evidencia las funciones y roles de los actores

EL AVA posee $y$ evidencia el rol del estudiante

EL AVA posee $y$ evidencia el rol del tutor

El AVA favorece la comunicación entre los participantes

EI AVA tiene un lenguaje académico adecuado a los estu

$5(20-18) / 4(17-14) / 3(13-9) / 2(8-5) / /(1-5)$

\section{$\square$ : Estrategia y contenidos}

El AVA está basado una estrategia y técnica didáctica

EL AVA posee una secuencia didáctica y co $\square$ erencia con la intención pedagógic

Es pertinente la temática al nivel educativo

Los temas centrales tienen más relevancia dentro del AVA y están destacados

Los contenidos del AVA corresponden al contenido programático

$5(20-18) / 4(17-14) / 3(13-9) / 2(8-5) / /(1-5)$

\section{Observaciones}

El aprendizaje está $\square$ avorecidqor el aprendizaje signi $\square$ icativo se evidencia en las actividades acad $\square$ micas $\square$ as $\square$ como el tema es pertinenlas necesidades de capacitación de los participantes (docentes).

\begin{tabular}{|c|c|}
\hline E: Actividades acad $\square$ micas y evaluación & Puntos \\
\hline Las actividades académicas del AVA mencionan el objetivo de aprendizaje & 4 \\
\hline $\begin{array}{l}\text { El AVA menciona la dinámica de la actividad } \square \text { poseenna conducta de entrada e instrucciones } \\
\text { claras para su desarrollo }\end{array}$ & 4 \\
\hline $\begin{array}{l}\text { El AVA menciona cómo se van a evaluar las actividades académicas y corresponden a los } \\
\text { contenidos y al material dise } \square \text { ado para el curso }\end{array}$ & 4 \\
\hline La evaluación es co $\square$ erente con el contenido del cuso & 4 \\
\hline El AVA utiliza elementos que promueven la autoevaluación $\square$ coevaluación $\bigvee$ leteroevaluación & 2 \\
\hline $5(20-18) / 4(17-14) / 3(13-9) / 2(8-5) / 1(1-5)$ & $\mathbf{I} \square$ \\
\hline
\end{tabular}

\begin{tabular}{|c|c|}
\hline F: Integración de las TI $\square$ & Puntos \\
\hline Los recursos educativos utilizados justifican la ense $\square$ anza detema & 4 \\
\hline El uso de la tecnolog $\square$ a apoya de manera óptima las estrategias de ense $\square$ anza $\square \square$ arris $\square \square$ & $\square \square[\square$ \\
\hline $\begin{array}{l}\text { La selección de tecnolog } \square a \square s \square \text { es ejemplar con respecto a los objetivos curriculares y las } \\
\text { estrategias de ense } \square \text { anza } \square \square \text { arris } \square \square \square \square \square\end{array}$ & 4 \\
\hline El uso de la tecnolog $\square$ a apoya de manera óptima las estrategias de ense $\square$ anza $\square \square$ arris $\square \square$ & $\square \square[\square$ \\
\hline $\begin{array}{l}\text { Dontenido Estrategias de ense } \square \text { anza tecnolog } \square \text { oe articulan completamente entre s[dentro } \\
\text { de la planificación didáctica } \square \square \text { arris } \square \square \square \square \square \square\end{array}$ & 4 \\
\hline $5(20-18) / 4(17-14) / 3(13-9) / 2(8-5) / /(I-5)$ & 20 \\
\hline
\end{tabular}

\begin{tabular}{|c|c|}
\hline$\square: \square$ alidad y pertinencia & Puntos \\
\hline Aspectos técnicos del AVA $\square$ basado en $\square$ errano $\square$ et al $\square$ sf $\square \square \square$ & 4 \\
\hline$\square$ istribución y calidad de contenidos $\square$ basado en $\square$ errano $\square$ et al $\square$ sf $\square \square \square$ & 4 \\
\hline Aspectos pedagógicos y motivacionales $\square$ basado en $\square$ errano $\square$ et al $\square$ sf $\square \square \square$ & 4 \\
\hline Valoración global y contextual del AVA $\square$ basado en $\square$ erranet $[a l ~ \square s \square \square \square$ & 4 \\
\hline El AVA maneja adecuadamente los derec $\square$ os de autor & 4 \\
\hline $5(20-18) / 4(17-14) / 3(13-9) / 2(8-5) / 1(1-5)$ & 20 \\
\hline
\end{tabular}

\begin{tabular}{|c|c|}
\hline $\begin{array}{c}\text { Parámetros } \\
\text { de evaluación }\end{array}$ & Puntos \\
\hline A & 19 \\
\hline B & 18 \\
\hline$\square$ & 19 \\
\hline$\square$ & 19 \\
\hline $\mathbf{E}$ & 18 \\
\hline $\mathbf{F}$ & 20 \\
\hline$\square$ & 20 \\
\hline$\square$ otal puntos & 133 \\
\hline
\end{tabular}

\section{Observaciones}

Los recursos son adecuados ya $\square$ uœl curso se realiza de manera virtual $\square$ s $\square$ mismo los recursos propuestos llevan a $\square$ uœl estudiante e $\square$ plorede lo $\square$ uœs capaz de investigar en su entorno para solucionar un problema $\square$ potencializando sus conocimientos previos.

Los criterios de evaluación corresponden a lo consignado en el contenido programático y evalúan lo $\square$ ue está en los contenido $\square$ sin embargo $\square$ se deben evidenciar las actividades de heteroevaluación y coevaluación.

EJE 2013

EJE 2013

Tabla 3. Evaluación del AVA para vincular el M-Learning a procesos de enseñanza-aprendizaje en instituciones de Educación Superior

e implementado. Asimismo, las actividades del AVA dan cuenta y enuncian cómo se van a evaluar en coherencia con las sesiones, promoviendo la autoevaluación; aunque es importante resaltar que el AVA debe potenciar las actividades de heteroevaluación y coevaluación donde se involucre en el proceso de evaluación a todos los participantes, lo cual indica que se deben incluir actividades en el AVA que cumplan con este criterio.

Las instrucciones en el AVA están dadas al inicio del curso y se publican en el tablón de noticias. Los temas tratados se desarrollan de acuerdo con lo planteado en el contenido programático. El material de consulta está en PDF y en enlaces. El AVA ofrece tutoriales y foros de dudas para los inconvenientes técnicos que se presentan en su desarrollo, ofreciendo así una variedad de recursos en la plataforma.

Lo anterior demuestra que la evaluación educativa es un importante instrumento para el mejoramiento continuo en busca de la calidad del proceso enseñanza-aprendizaje de los ambientes virtuales de aprendizaje, a partir de la interpretación y análisis de los aspectos evaluados. 
Hacia una propuesta para evaluar ambientes virtuales de aprendizaje (AVA) en Educación Superior

\section{Conclusiones}

Partiendo de la premisa que la evaluación educativa es un instrumento importante para el mejoramiento continuo en busca de la calidad del proceso enseñanza-aprendizaje, se encontró que los resultados a partir de la evaluación de AVA, no deben entenderse como términos absolutos o verdades finales, sino, por el contrario, los fenómenos y/o resultados de la evaluación educativa deben ser interpretados y comprendidos desde las características propias en las que se desarrollan los objetivos de los ambientes de aprendizaje, que como tal son variables; también deben ser interpretados en términos disciplinarios, desde la práctica educativa pasando por la didáctica y la pedagogía.

Es importante recordar que el fin de todo proceso evaluativo, no es indicar si algo está bien o mal, sino dar orientaciones que sirvan para ajustar los procesos que se desarrollan, ya sea, a través de información que permita a los autores del AVA reflexionar sobre los resultados y a partir de éstos realizar los ajustes que consideren necesarios, o a través de la identificación de los puntos débiles dentro del diseño del AVA. Entendiendo por puntos débiles, aquellos elementos, bien sea recursos educativos, actividades o secuencias, en donde no es clara la forma como estos elementos pueden contribuir a los objetivos planteados por el AVA.

Finalmente, la comprensión de resultados que se arrojan a través de la rúbrica para la evaluación de AVA, verificar la eficacia de un programa o sistema para apoyar la toma de decisiones oportunas, lo cual puede aportar verdaderamente al avance y al mejoramiento de los programas de educación virtual en Colombia.

\section{Referencias}

Boude Figueredo, O. (Producer) (2012). Estrategias Didácticas. mmap (Universidad de la Sabana). [MMAP]. Disponible en http://virtual.unisabana.edu. $\mathrm{co} /$ course/view.php?id $=2845 \&$ topic $=4$

Boude Figueredo, O. (Producer) (2012). Concepción del AA. mmap (Universidad de la Sabana). [MMAP]. Disponible en http://virtual.unisabana.edu.co/course/ view.php?id $=2845 \&$ topic $=4$

Boude Figueredo, O. (Producer) (2012). Diseño de un AA. mmap (Universidad de la Sabana). [MMAP]. Disponible en http://virtual.unisabana.edu.co/course/ view.php?id $=2845 \&$ topic $=4$

Boude Figueredo, O. (2011) Pediatic: desarrollo de competencias en TIC a través del aprendizaje por proyectos. Educ Med Super [online]. 2011, vol.25, n.2 [citado 2014-04-10], pp. 116-124. Disponible en: $\quad<$ http://scielo.sld.cu/scielo.php?script=sci arttext\&pid=S0864-21412011000200009\&lng=es\&nr $\mathrm{m}=\mathrm{iso}>$. ISSN $0864-2141$

Boude Figueredo, O. \& Jimenez, J. (2016, En prensa) Framework for Design of Mobile Learning Strategies. En Zhang, Yu (Aimee) (Ed.) Handbook of Mobile Teaching and Learning. New York, EE.UU: Springer reference

Cabero, J. (2002). Análisis, selección y evaluación de medios audiovisuales didácticos.

Disponible en http:/tecnologiaedu.us.es/revistaslibros/ qurricul.html 
Cerrano, M., Fulgueira, S. \& Gómez, D. (sf). Instrumento para Medir la Calidad de un Material Didáctico Multimedia para Educación a Distancia. [Versión en línea]. Disponible en http://virtual.unisabana. edu.co/pluginfile.php/194902/mod_assign/intro/2\%20Cerrano-Fulgueira-Gomez-Instrumento. pdf

Harris, J., Grandgenett, N., \& Hofer, M. (2010). Rúbrica de evaluación de integración de tecnología. Disponible en http://encuentro.educared.org/page/taxonomiasjudi-harris

Lukas, J. F. Y Santiago, K. (2009) Evaluación Educativa. Segunda edición. Madrid: Alianza Editorial. 332 Págs. Capítulo 1: Naturaleza de la Investigación y Evaluación en Educación. Págs. 13 a 61.

Otálora Sevilla, Y. (2010). Diseño de espacios educativos significativos para el desarrollo de competencias en la infancia. CS, No.5, pp. 71-96. ISSN 2011-0324 Retrieved from http://virtual.unisabana.edu.co/course/ view.php?id=2845

UNESCO, (2010). Informe mundial sobre el aprendizaje y la educación de adultos (ISBN 978-92-820-3074-5 (electrónica) Instituto de la UNESCO para la Educación a lo Largo de Toda la Vida). Feldbrunnenstrasse 58 20148 Hamburgo Alemania: UNESCO. 